\title{
Adrenal venous sampling as a diagnostic procedure for primary hyperaldosteronism: experience from a tertiary referral centre
}

\author{
Victoria Salem, ${ }^{1}$ Thomas G. Hopkins, ${ }^{1}$ Heba El-Gayar, ${ }^{1}$ Sagen Zac-Varghese, ${ }^{1}$ \\ Anthony P. Goldstone, ${ }^{1}$ Jeannie F. Todd, ${ }^{1}$ Waljit Dhillo, ${ }^{1}$ Benjamin C.T. Field, ${ }^{1}$ \\ Niamh Martin, ${ }^{1}$ Emma Hatfield, ${ }^{1}$ Mandy Donaldson, ${ }^{2}$ Fausto Palazzo, ${ }^{3}$ \\ Karim Meeran, ${ }^{1}$ James Jackson, ${ }^{4}$ Tricia $\operatorname{Tan}^{1,2}$
}

Departments of ${ }^{1}$ Endocrinology, ${ }^{2}$ Chemical Pathology, ${ }^{3}$ Surgery, ${ }^{4}$ Radiology, Imperial College Healthcare NHS Trust, Hammersmith Hospital, Du Cane Road, London, U.K.

\begin{abstract}
CONTEXT: Adrenal vein sampling (AVS) is recommended in all patients with hyperaldosteronism to whom surgery would be offered if the results indicated unilateral hypersecretion. OBJECTIVE: To assess the performance of AVS against radiological findings and to evaluate the Endocrine Society's Practice Guidelines for diagnostic cut-offs. PATIENTS: Retrospective study of 41 patients with hyperaldosteronism who underwent both AVS and computed tomography (CT) imaging. RESULTS: CT and AVS results were concordant in $73.7 \%$. Unilateral lesions on CT had a greater positive predictive value $(85 \%)$ than non-unilateral lesions $(50 \%)$. In patients with subsequently confirmed adrenal adenomas, a lateralisation ratio $>2$ when comparing cortisol-corrected aldosterone ratios from the affected versus unaffected side was $100 \%$ sensitive. Patients who were managed surgically experienced significant reductions in blood pressure and medication burden and $46 \%$ were cured. CONCLUSIONS: AVS is important in establishing unilateral or bilateral adrenal secretion of aldosterone in patients with primary hyperaldosteronism. However, it may not be essential for the work-up in patients below the age of 40, in whom adrenal incidentalomas adrenal incidentalomas are known to be rarer, and a unilateral lesion on CT therefore has a greater positive predictive value.
\end{abstract}

Key words: Adrenal cortex, Adrenal cortex function tests, Adrenalectomy, Aldosterone, Hyperaldosteronism

\section{INTRODUCTION}

Primary hyperaldosteronism is an important and

Address for correspondence:

Tricia Tan, Tel.: +44 20 83838242, Fax: +44 2083838142 , e-mail: t.tan@imperial.ac.uk

Received 16-09-11, Revised 09-11-11, Accepted 17-01-12 potentially curable form of hypertension, accounting for over $10 \%$ of cases of resistant hypertension. ${ }^{1}$ Distinguishing unilateral from bilateral adrenal disease and hence selecting those patients suitable for curative surgical management requires a multidisciplinary approach. The pre-operative assessment 
of all patients with primary hyperaldosteronism by adrenal vein sampling (AVS) has been a debatable issue. ${ }^{2}$ The AVS procedure is invasive, requires a skilled operator and is therefore not widely available. However, its routine use in the workup of patients with primary hyperaldosteronism is advocated due to the high incidence of misleading adrenal cross-sectional imaging findings. ${ }^{3-5}$

Due to the range of different AVS techniques across centres there are no generally accepted AVS diagnostic cut-offs. The Endocrine Society guidelines suggest that a cortisol-corrected aldosterone ratio of $>2$ from high side to low side $\left(\mathrm{A}: \mathrm{C}_{\text {high }} / \mathrm{A}: \mathrm{C}_{\text {low }}\right.$ or lateralisation ratio), in the absence of cosyntropin use, indicates unilateral disease. When cosyntropin is infused during the AVS procedure, a lateralisation ratio $>4$ indicates unilateral disease and $<3$ is consistent with bilateral hypersecretion. Patients with ratios between 3 and 4 may have either condition and should be assessed alongside other clinical and radiological parameters. Unilateral hypersecretion can alternatively be concluded when the ratio of the cortisol-corrected aldosterone ratios between the affected gland and a peripheral vein (usually the inferior vena cava) is higher than 2.5 in the setting of suppressed aldosterone secretion from the contralateral gland (A: $\mathrm{C}_{\text {high }} / \mathrm{A}: \mathrm{C}_{\mathrm{IVC}}>2.5$ and $\left.\mathrm{A}: \mathrm{C}_{\mathrm{low}} / \mathrm{A}: \mathrm{C}_{\mathrm{IVC}}<1\right){ }^{5}$

Assessment of adequate adrenal vein catheterisation can often be achieved visually by the radiologist at the time of the procedure. The biochemical confirmation of successful catheterisation can also be validated using a strict criterion where the ratio between the cortisol values in the adrenal vein and a peripheral vessel $\left(\mathrm{C}_{\text {adrenal }}: \mathrm{C}_{\mathrm{IVC}}\right)$ is greater than $3 .^{6}$

We present findings from an audit of 41 patients in our unit who had a confirmed biochemical diagnosis of primary hyperaldosteronism and went on to have both adrenal computed tomography (CT) imaging and AVS. Our unit currently offers AVS to all patients with confirmed primary hyperaldosteronism who would be eligible for surgery if the diagnosis of unilateral secretion is confirmed. We examine our concordance rate between AVS and CT scan and discuss instances of discordant results in more detail. We present patient characteristics and outcomes stratified by whether they underwent surgical or medical management.
We have also retrospectively analysed the guideline biochemical cut-offs from the AVS samples.

\section{METHODS}

\section{Data collection}

This is a retrospective study of all adrenal vein sampling procedures performed in our unit since 2000. The study was approved by the institutional audit committee. The diagnosis of primary hyperaldosteronism was made in all patients by measuring a plasma aldosterone/renin ratio ( $>2000$, where aldosterone is measured in $\mathrm{pmol} / \mathrm{L}$ and plasma renin activity in $\mathrm{pmol} / \mathrm{mL} / \mathrm{h}$ ). Since 2009 , this diagnosis has also been confirmed with a salt-loading test using intravenous $0.9 \%$ saline to confirm non-suppression of aldosterone levels. Forty-one patients were identified for whom an analysable set of data could be extracted. Age at diagnosis, sex, blood pressure and serum potassium at presentation were recorded. Where possible, we collected information about pretreatment blood pressure (BP) and serum potassium $\mathrm{K}^{+}$concentrations from the referring practitioner. Antihypertensive medication being taken at the time of each BP recording and serum potassium measurement was noted. If the patient underwent surgery, the histology report of the removed adrenal gland was obtained. Surgically treated patients were deemed to have been cured if they were able to discontinue all antihypertensive medications in the long term. Medically-managed patients were deemed to have been successfully treated after pharmacological optimisation of blood pressure and serum electrolytes in the Endocrine Clinic. The requirement for potassium supplementation at any point during the patients' management was recorded. Follow-up information about $\mathrm{BP}$, serum $\mathrm{K}^{+}$and medications was taken from the medical records for the immediate post-treatment period and by contacting the patients' primary care physicians.

\section{Adrenal venous sampling protocol}

In our unit all AVS procedures have been performed by a single, experienced operator (JJ) since 2005 ; in the study period before this, the procedure was performed by one of three experienced interventional radiologists. Medications discontinued prior to the procedure are: aldosterone antagonists 
and oestrogens ( 6 weeks before), diuretics (4 weeks before), ACE inhibitors and angiotensin II receptor blockers ( 2 weeks before), dihydropyridine-based calcium channel antagonists, sympathomimetics and beta-blockers ( 1 week before). Where necessary for blood pressure control, these agents are replaced with prazosin, doxazosin or verapamil. Patients are placed on unrestricted salt intake prior to admission and oral potassium supplements are administered to achieve a serum $\mathrm{K}^{+}$concentration of $>3.5 \mathrm{mmol} / \mathrm{L}$. A catheter is inserted percutaneously via the femoral vein, the adrenal veins are selectively cannulated under X-ray guidance and venography is performed to confirm a satisfactory catheter position. Cosyntropin is not given. Samples are obtained from each adrenal vein in turn for cortisol and aldosterone. If the radiologist is in any doubt about the catheter position within the cannulated vein during aspiration of blood, a second, and sometimes a third, sample is obtained from that vessel; only one sample is taken if the catheter position is felt to be ideal. The sample containing the highest cortisol value is used for subsequent analysis. A peripheral sample is also taken from the inferior vena cava below the level of the renal veins. Interpretation of the results is undertaken in a multidisciplinary setting involving endocrine surgeons, radiologists and endocrinologists. We take cortisol-corrected aldosterone ratios into consideration, relying on a lateralisation ratio $\left(\mathrm{A}: \mathrm{C}_{\text {high }} / \mathrm{A}: \mathrm{C}_{\text {low }}\right)>2$ (alongside evidence of contralateral gland suppression), but cut-offs are not strictly adhered to because we use a diagnostic approach that also takes into account imaging results and clinical presentation.

\section{CT interpretation}

All patients included in this series underwent multidetector computed tomography (MDCT) of the adrenal glands without intravenous contrast medium, and multiplanar reformatted images were obtained from the volumetric data that were acquired. We used the radiologist's formal report at the time of the scan to categorise the imaging result into unilateral adrenal lesion (UAL - left or right), bilateral adrenal lesion (BAL) or no adrenal lesion (NAL). CT and AVS findings were judged to be concordant if CT showed a UAL and AVS lateralised to the same side or if CT showed either BAL or NAL and AVS confirmed bilateral hypersecretion.

\section{Assays}

Aldosterone was assayed using a coated tube direct radioimmunoassay (Siemens, UK). Plasma renin was assayed using an 'in house' enzyme kinetic assay, an indirect measurement of renin activity in vitro. The accumulation of angiotensin I from serum incubated at $37^{\circ} \mathrm{C}$ in the presence of an ACE inhibitor is assessed using a radioimmunoassay for angiotensin $\mathrm{I}$. Until March 2006, cortisol was measured using the Nichols Advantage competitive chemiluminescence immunoassay. Due to the withdrawal of the Nichols Advantage assay, from March 2006 onward cortisol was measured using the Immulite 2000 assay (Siemens, UK).

\section{Statistics}

Data were analysed by Prism (Version 5.0, GraphPad Software Inc, San Diego, CA, USA). All measurements are given as mean \pm SEM. Unpaired $t$ tests were used to compare $\mathrm{BP}$ and serum $\mathrm{K}^{+}$differences between the surgically and medically managed groups, after confirmation of the normality of data distribution using the d'Agostino and Pearson test. Paired t tests were used to compare pre-and post- treatment measurements. A Mann-Whitney test was used to compare the change in anti-hypertensive requirements (number of drugs taken) pre- and post-treatment between the surgically and medically managed groups. For all cases, values of $\mathrm{p}<0.05$ were considered significant.

\section{RESULTS}

Table 1 summarises the biochemical, imaging and, if surgically treated, histological results for the cohort of patients included in this series.

\section{Concordance analysis}

AVS was judged to have been non-diagnostic (either at the time of attempted adrenal cannulation or on subsequent biochemical interpretation) in 2 out of $41(4.8 \%)$ cases. In both cases, the non-diagnostic AVS procedure was performed before the introduction of the single-operator policy in 2005. Failure was in both cases due to inability to cannulate and sample from the right adrenal vein, which is technically the more difficult for a number of reasons including: its small calibre, its short length and the fact that it drains directly into the IVC, which is a large calibre and col- 


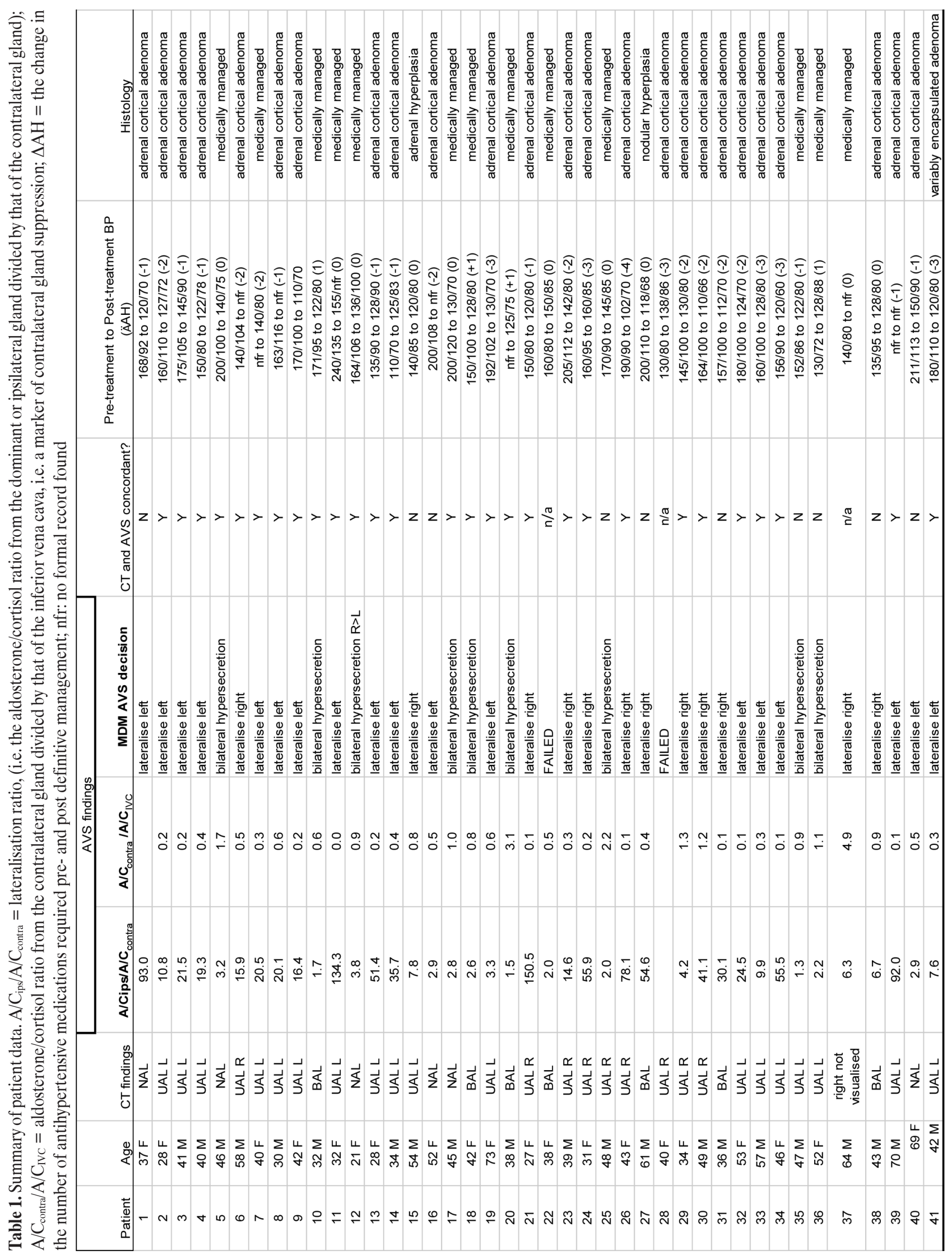


lapsible vessel in which fine catheter manipulations are more difficult. ${ }^{7}$ There were no procedure-related complications. In one further case, CT was unable to visualise the adrenal glands adequately due to large renal cysts. Of the remaining 38 cases, CT and AVS findings were concordant in 28 patients $(73.7 \%)$. A breakdown of how the imaging results compared with the AVS result is shown in Table 2.

\section{Surgery Group}

Twenty-eight out of the 41 patients underwent a unilateral laparoscopic adrenalectomy. Pre-operative AVS was non-diagnostic in one patient, but in this case adrenal CT showed a UAL, which was confirmed to be an adenoma at removal. The histology report of the 28 surgically treated patients confirmed an adrenal cortical adenoma in all but two instances, where the findings were of nodular hyperplasia. In both of these cases, AVS had clearly indicated unilateral secretion (Table 1, case numbers 15 and 27), although in both cases the AVS and CT findings also happened to be discordant (UAL on the opposite side in one and BAL in the other). As might be predicted, neither of these patients experienced a surgical cure, requiring the same number of antihypertensives post-operatively (two each), albeit achieving better BP control.

The mean age of patients undergoing surgery was $44.7 \pm 2.34$ years and $44 \%$ were male. Mean BP $(\mathrm{mmHg})$ at presentation and minimum 3 months post-operatively were $162 \pm 5.1 / 97 \pm 2.3$ and $127 \pm 2.7$ $175 \pm 3.4$ respectively $(\mathrm{p}<0.001$ for the observed postoperative reductions in both systolic and diastolic BP - Figure 1). Mean pre- and post-operative serum $\mathrm{K}^{+}$measurements $(\mathrm{mmol} / \mathrm{L})$ were $2.96 \pm 0.12$ and $4.4 \pm 0.09(\mathrm{p}<0.001$, Figure 2). Pre-operatively, $60 \%$

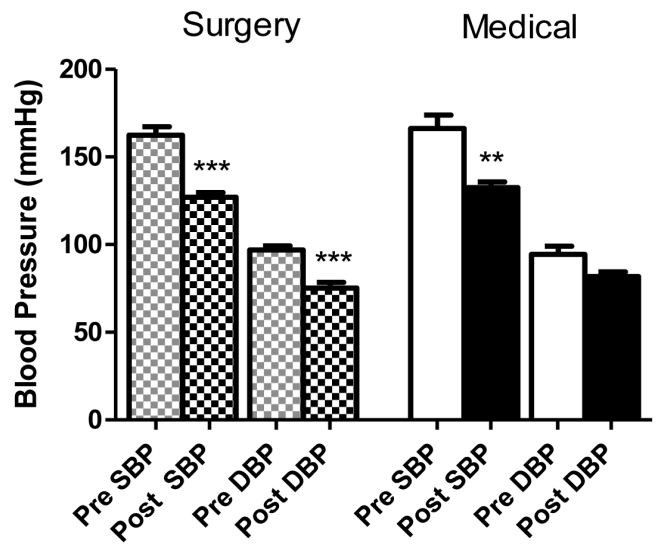

Figure 1. Pre- and post-treatment blood pressures (BP) by group. The systolic BP (SBP) and diastolic BP (DBP) recorded pre and post-unilateral adrenalectomy (surgery: chequered columns, $n=28$ ) are plotted on the left-hand side. SBP and DBP recorded pre and post-pharmacological optimisation of $\mathrm{BP}$ are plotted on the right hand side (medical: solid columns, $n=10$ ). Error bars denote the S.E.M. ${ }^{* *}=p<0.010$ and $^{* * *}=p<0.001$ (paired t-test compared to the pre-treatment value).

of these patients required potassium supplementation, the average requirement being $79 \mathrm{mEq}$ per day. No patient required supplements post-operatively. After surgery, the mean number of anti-hypertensive medications required by each patient also fell from $2.7 \pm 0.25$ to $1.1 \pm 0.26$. Figures $3 a$ and $3 b$ highlight the changes in antihypertensive requirements with surgical versus medical treatment. Overall, after surgical treatment, 13 patients $(46 \%)$ were normotensive off all medications and were therefore deemed to have been cured. One surgical patient's post-operative recovery was complicated by a wound infection. In this case, the adrenalectomy had been converted from laparoscopic to open due to adhesions from previous abdominal surgery.

Table 2. Comparison of Imaging Results with AVS

\begin{tabular}{|c|c|c|c|c|c|}
\hline \multirow[b]{2}{*}{ CT Result } & & \multicolumn{3}{|c|}{ AVS Result } & \multirow[b]{2}{*}{$\begin{array}{c}\text { Accurate CT } \\
\%(\mathbf{n} / \mathbf{n})\end{array}$} \\
\hline & & $\begin{array}{l}\text { Unilateral right } \\
\text { Hypersecretion }\end{array}$ & $\begin{array}{c}\text { Unilateral left } \\
\text { Hypersecretion }\end{array}$ & $\begin{array}{c}\text { Bilateral } \\
\text { Hypersecretion }\end{array}$ & \\
\hline \multirow[t]{2}{*}{ Unilateral } & Unilateral right (UAL R) & 7 & 0 & 1 & \multirow[t]{2}{*}{$84.6 \%(22 / 26)$} \\
\hline & Unilateral left (UAL L) & 1 & 15 & 2 & \\
\hline \multirow[t]{2}{*}{ Not unilateral } & Bilateral abnormality (BAL) & 3 & 0 & 3 & \multirow{2}{*}{$\begin{array}{c}50 \% \\
(6 / 12)\end{array}$} \\
\hline & No abnormality detected (NAL) & 0 & 3 & 3 & \\
\hline \multicolumn{2}{|c|}{$\begin{array}{l}\text { AVS result concordant with CT } \\
\%(\mathrm{n} / \mathrm{n})\end{array}$} & $63.6 \%(7 / 11)$ & $83.3 \%(15 / 18)$ & $66.7 \%(6 / 9)$ & $\begin{array}{c}\text { Overall } \\
73.7 \%(28 / 38)\end{array}$ \\
\hline
\end{tabular}




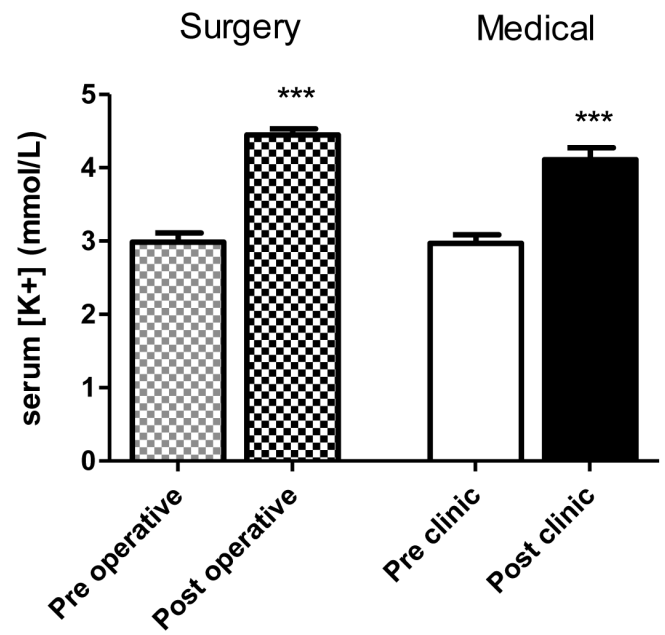

Figure 2. Pre- and post-treatment serum potassium levels by treatment group. Serum potassium levels in $\mathrm{mmol} / \mathrm{L}$ pre- and post-unilateral adrenalectomy (surgery: chequered columns, $\mathrm{n}=28$ ) are plotted on the left-hand side, and pre- and postpharmacological optimization of blood pressure are plotted on the right-hand side (medical: solid columns, $n=10$ ). Error bars denote the S.E.M. ${ }^{* * *}=\mathrm{p}<0.001$ (paired t-test compared to the pre-treatment value).

\section{Non-Surgery Group}

Thirteen patients underwent medical management alone. In two cases AVS suggested unilateral hypersecretion, which was supported by concordant imaging findings, but the patients ultimately refused surgery. In one further case AVS suggested unilateral secretion, but the adrenals could not be visualised on CT due to the presence of large renal cysts, and surgery was deemed inappropriate for the same reason. Analysis of patient characteristics in the medically-managed group is therefore limited to the remaining 10 patients who were diagnosed with bilateral hypersecretion on AVS.

The mean age of the patients in this group was $40.9 \pm 2.88$ years and $60 \%$ were male. Mean blood pressures at presentation and following treatment opti- misation in the Endocrine clinic were 166 $\pm 7.6 / 94 \pm 4.8$ $\mathrm{mmHg}$ and $132 \pm 3.1 / 82 \pm 2.6 \mathrm{mmHg}$ respectively $(\mathrm{p}<0.010$ for the post-optimisation reduction in systolic BP, whilst the observed reduction in diastolic BP did not reach statistical significance in this smaller sample of medically-managed patients). Mean pre- and post-treatment serum potassium measurements were $2.97 \pm 0.11 \mathrm{mmol} / \mathrm{L}$ and $4.1 \pm 0.16 \mathrm{mmol} / \mathrm{L}(\mathrm{p}<0.001)$ respectively (Figures 1 and 2). Fifty percent (50\%) of patients required potassium supplementation, the average requirement being $54 \mathrm{mEq}$ per day. However, after treatment optimisation in the Endocrine clinic, no patient required long-term potassium supplementation. From referral to discharge from the Endocrine clinic, the mean number of antihypertensive medications taken by each patient remained unchanged (2.5 to 2.8). All took an aldosterone antagonist: the average dose of spironolactone in the long term was $120 \mathrm{mg}$ per day. Three patients had to be switched to an alternative aldosterone antagonist due to side effects, either eplerenone or amiloride.

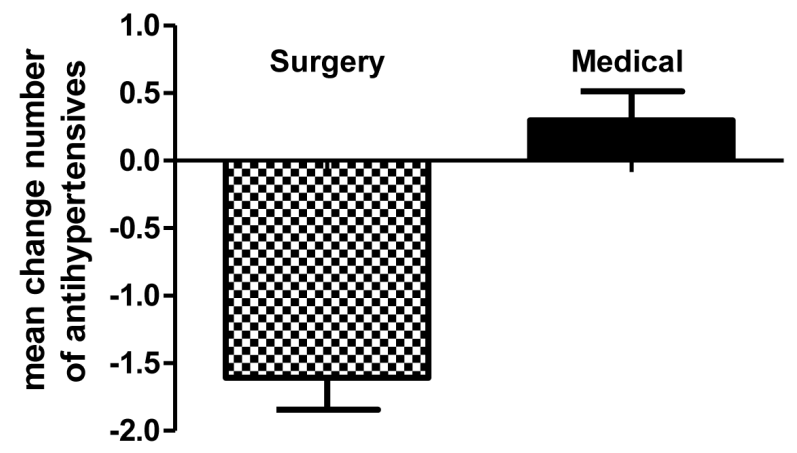

Figure 3b. Mean change in the number of anti-hypertensive medications after definitive treatment (surgery: chequered columns, $n=28$ vs medical management: solid columns, $n=10$ ). Error bars denote the S.E.M.

$\mathrm{p}<0.001$ (Mann-Whitney test comparing the two treatment groups)

\begin{tabular}{|l|c|c|c|c|c|c|c|c|c|}
\cline { 2 - 10 } \multicolumn{1}{c|}{} & \multicolumn{8}{c|}{ Change in number of anti-hypertensives required } & \multicolumn{1}{c|}{} \\
\cline { 2 - 11 } & $\mathbf{- 4}$ & $\mathbf{- 3}$ & $\mathbf{- 2}$ & $\mathbf{- 1}$ & $\mathbf{0}$ & $\mathbf{+ 1}$ & $\mathbf{+ 2}$ & $\mathbf{+ 3}$ & TOTAL \\
\hline Surgically managed group & 1 & 6 & 8 & 9 & 3 & 0 & 1 & 0 & 28 \\
\hline Medically managed group & 0 & 0 & 1 & 1 & 7 & 4 & 0 & 0 & 10 \\
\hline
\end{tabular}

Figure 3a. Contingency table showing the number of antihypertensive drugs taken by patients pre- and post-definitive treatment according to treatment group (surgical versus medical management). 
There were no differences in BP or serum $\mathrm{K}^{+}$at presentation between the patients diagnosed with unilateral versus bilateral disease. Average supplemental (oral) potassium requirements prior to optimal treatment were lower in the medically-managed groups (i.e. those diagnosed with bilateral adrenal hypersecretion), but this did not reach statistical significance (Figure 4).

\section{Comparison with Endocrine Society Clinical Practice Guidelines for Diagnostic Cut-off Values in AVS}

We examined our AVS results against the diagnostic criteria in the Endocrine Society's guidelines. A full set of AVS data and the histopathological examination was available for 26 surgically treated patients, of whom 24 had adenomata and 2 had nodular hyperplasia (as previously described). There were 9 patients with a full set of AVS data diagnosed with bilateral hypersecretion. Using these groups of patients, and assuming that histological confirmation of adenoma was the gold standard for the diagnosis of unilateral disease (with the caveat that no such gold standard exists for the confirmation of bilateral disease), then a $\mathrm{A}: \mathrm{C}_{\text {high }} / \mathrm{A}: \mathrm{C}_{\text {low }}$ (lateralisation ratio) $>2$ was $100 \%$ sensitive at diagnosing unilateral disease, but only $27 \%$ specific (although with a negative predictive value of $100 \%)$. The alternative diagnostic criterion of $\mathrm{A}: \mathrm{C}_{\text {high }} / \mathrm{A}: \mathrm{C}_{\mathrm{IVC}}>2.5$ and $\mathrm{A}: \mathrm{C}_{\text {low }} / \mathrm{A}: \mathrm{C}_{\mathrm{IVC}}<1$ was associated with a sensitivity of $71 \%$ and specificity of

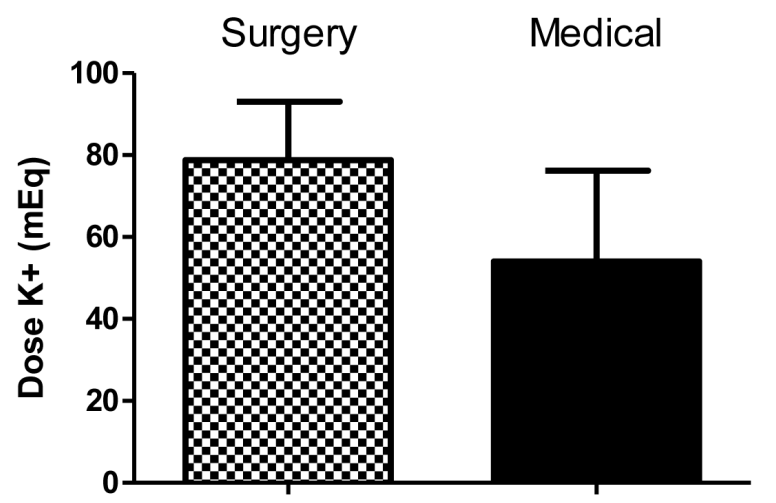

Figure 4. Daily doses of oral supplemental potassium taken before definitive treatment. Daily doses plotted in $\mathrm{mEq} /$ day. Surgical group on left (chequered column). Medical group on right (solid column). No patient in either group required long-term oral potassium supplementation after definitive management had been implemented.
$72 \%$. Confirmation of adrenal vein catheterisation with $\mathrm{C}_{\text {adrenal }} / \mathrm{C}_{\mathrm{IVC}}>3$ was achieved in all but 2 instances (where the values were 2.7 and 2.8).

\section{DISCUSSION}

This study of 41 patients who have been treated for primary hyperaldosteronism supports the important diagnostic role of AVS. Our concordance rate between AVS and imaging findings of $73.7 \%$ is slightly better than the $62.2 \%$ reported in a meta-analysis of other case series, ${ }^{5}$ and highlights examples where imaging alone can correctly identify unilateral disease, as well as examples where, if used alone, it could be harmfully misleading. Amongst our patients, the following errors may have occurred had the decision to operate been based on radiological findings alone:

Five cases (12\%) would have been inappropriately given life-long medical therapy. Instead, these cases were operated on with resulting improvement in serum potassium levels post-operatively and reduction in medication burden in all, and cure in one case.

In one case $(2 \%)$, the non-secreting adrenal would have been removed erroneously. Instead, the secreting adrenal was removed, with resulting improvement in potassium levels post-operatively.

In three cases (7\%), unilateral adrenalectomy would have been performed to treat bilateral hypersecretion. Whether this treatment is appropriate remains a matter of debate, although one series has been published which suggests some benefit. ${ }^{8}$

STARD guidelines for the evaluation of biochemical diagnostic tests ${ }^{9}$ suggest that a diagnostic test should be evaluated in comparison with a gold standard. There are several reasons why the assessment of AVS as a diagnostic tool can never be entirely complete in this way:

AVS is itself often referred to as the gold standard for the differential diagnosis of adrenal disease. However, whilst AVS may indeed be more accurate than imaging, it is technically demanding, with an operatordependent failure rate, and can produce equivocal results with imperfect sensitivity and specificity, as shown here and in other retrospective studies. ${ }^{3,6,10}$

If a patient is referred for surgery, histopathologi- 
cal confirmation of an adrenal cortical adenoma may be cited as definitive evidence of unilateral disease. However, the criteria for the histopathological diagnosis of adrenal adenoma are not clearly defined and there are varying degrees of overlap between pathological morphologies - for example mixed histology, "dominant" nodules or poorly circumscribed adenomata. ${ }^{11,12}$ Occasionally, the post-operative clinical and biochemical course of the patient is required to assess whether a cure has been achieved.

Even if histological findings are to be taken into account, the performance of AVS can never fully be evaluated in patients diagnosed with bilateral disease who do not undergo adrenal surgery.

Thus, there remains an inclination to limit the use of AVS to a more highly selected cohort. Comparison of our AVS and CT findings (Table 2) reveal the possibility that unilateral lesions on $\mathrm{CT}$ do indeed have a greater positive predictive value (85\%) compared with non-unilateral lesions (50\%). It has previously been suggested that younger patients presenting with a unilateral lesion on CT and hypokalaemia pose a lesser diagnostic challenge and may proceed directly to surgery without confirmatory AVS. ${ }^{5,13}$ We have reported here 3 patients with unilateral lesions on CT $<1 \mathrm{~cm}$ but discordant evidence of bilateral hypersecretion on AVS, all of whom were aged over 40 years (47, 48 and 56). One other 57-year old patient had a bulky left adrenal reported on CT but AVS lateralised to the right. In retrospect, these 4 patients would have been highlighted as having potentially inaccurate CT results based on their age $(>40)$ and nodule size, and could have been offered AVS at that stage. In our total cohort of 41 patients, 16 were aged under 40 years. Eleven of those under 40 years of age had unilateral lesions with concordant AVS findings. One declined surgery but histological confirmation of adenoma was obtained for the other ten. Thus, in our cohort, no diagnostic errors would have occurred had all patients under the age of 40 years with unilateral CT lesions been directed to surgery without AVS.

We have also demonstrated that, where AVS findings (with or without radiological agreement) strongly point to unilateral disease, surgery is clearly the best option if acceptable to the patient. Although in our follow-up period ( 3 months-10 years), surgi- cal treatment did not confer improved BP control or electrolyte profile compared with best medical intervention, there was a reduced medication burden and around a $46 \%$ chance of cure. Aldosterone antagonists are associated with numerous and often intolerable side effects and must be used with contraception in women of childbearing age due to their teratogenic potential. Their long-term use, from the point of view of compliance and the cardiovascular consequences of imperfect aldosterone antagonism may also be a concern.

In the setting of bilateral adrenal hypersecretion, the diagnostic performance of AVS can never be conclusively tested since histological confirmation of hyperplasia in both glands is not possible when medical management is chosen. The situation is further complicated by reports of equivocal histological findings, including macronodules, in the removed adrenals of patients diagnosed with bilateral disease on AVS. ${ }^{11}$ Amongst our patients, there were two surgical cases where the histology appeared to be more in keeping with nodular adrenal hyperplasia than a discrete adenoma. As described earlier, both patients were not cured with surgery but achieved much improved blood pressure control on the same number of antihypertensives. On such tenuous grounds, we are unable to make further comment on unilateral adrenalectomy as a treatment option to ameliorate bilateral disease.

Whilst the case for the use of AVS in the work-up of patients with hyperaldosteronism is strong, there are, confusingly, numerous criteria for analysing the results. ${ }^{4-6}$ In our unit, where cosyntropin is not infused during the procedure, a lateralisation index of $>2$ was present in all cases of histologically confirmed adrenal adenomas, and in all but one of these cases there was evidence of contralateral gland suppression (defined as $\mathrm{A}: \mathrm{C}_{\text {low }}$ no greater than $\mathrm{A}: \mathrm{C}_{\mathrm{IVC}}$ ). The appropriate cut-off values for $\mathrm{A}: \mathrm{C}$ ratios in the group diagnosed with bilateral disease are less clear, possibly because this group may contain some patients with mild unilateral disease. Hence, when the AVS lateralisation ratio is slightly greater than 2 but with no evidence of contralateral suppression, other factors such as radiological findings need to be taken into account. Furthermore, due to variation in procedural techniques and biochemical analysis, each centre must validate its own diagnostic cut-offs. 
In summary, AVS should be considered in all patients with primary hyperaldosteronism who would be fit and prepared to undergo surgery if the findings were consistent with unilateral disease. However, it is reasonable to exercise discretion in terms of selection for AVS. Our findings cohere with recommendations that surgery may be considered without AVS in young patients with clear radiological evidence of a unilateral adrenal lesion. ${ }^{5,13}$ In other cases, patients would be likely to benefit from AVS performed by expert hands. Where the procedure is performed by a single experienced operator, patients can be reassured that AVS is safe and the failure rate is low. A multidisciplinary decision taking all data into consideration is then recommended with regards to the possibility of ameliorating hypertension with unilateral adrenalectomy versus life-long pharmacological treatment.

\section{ACKNOWLEDGEMENTS:}

\section{Dr Mike Scanlon, Department of Chemical Pathology, St Mary's Hospital.}

DrV Salem is the recipient of a Medical Research Council Clinical Research Training Fellowship.

No conflicting interests to declare.

\section{REFERENCES}

1. Douma S, Petidis K, Doumas M, et al, 2008 Prevalence of primary hyperaldosteronism in resistant hypertension: a retrospective observational study. Lancet 371 : 1921-1926.

2. Stewart PM, Allolio B, 2010 Adrenal vein sampling for Primary Aldosteronism: time for a reality check. Clin Endocrinol (Oxf) 72: 146-148.

3. Young WF, Stanson AW, Thompson GB, Grant CS, Farley DR, van Heerden JA, 2004 Role for adrenal venous sampling in primary aldosteronism. Surgery 136: 1227-1235.

4. Kempers MJ, Lenders JW, van Outheusden L, et al, 2009 Systematic review: diagnostic procedures to differentiate unilateral from bilateral adrenal abnormality in primary aldosteronism. Ann Intern Med 151: 329-337.

5. Funder JW, Carey RM, Fardella C, et al, 2008 Case detection, diagnosis, and treatment of patients with primary aldosteronism: an Endocrine Society clinical practice guideline. J Clin Endocrinol Metab 93: 32663281.

6. Mulatero P, Bertello C, Sukor N, et al, 2010 Impact of different diagnostic criteria during adrenal vein sampling on reproducibility of subtype diagnosis in patients with primary aldosteronism. Hypertension 55: 667-673.

7. Doppman JL, Gill JR, Jr, 1996 Hyperaldosteronism: sampling the adrenal veins. Radiology 198: 309-312.

8. Sukor N, Gordon RD, Ku YK, Jones M, Stowasser M, 2009 Role of unilateral adrenalectomy in bilateral primary aldosteronism: a 22-year single center experience. J Clin Endocrinol Metab 94: 2437-2445.

9. Bossuyt PM, Reitsma JB, Bruns DE, et al, 2003 Towards complete and accurate reporting of studies of diagnostic accuracy: the STARD initiative. BMJ 326: 41-44.

10. Harvey A, Kline G, Pasieka JL, 2006 Adrenal venos sampling in primary hyperaldosteronism: comparison of radiographic with biochemical success and the clinical decision-making with "less than ideal" testing. Surgery 140: 847-853.

11. Gordon RD, Stowasser M, Klemm SA, Tunny TJ, 1995 Primary aldosteronism-some genetic, morphological, and biochemical aspects of subtypes. Steroids 60: 35-41.

12. Boulkroun S, Samson-Couterie B, Dzib JF, et al, 2010 Adrenal cortex remodeling and functional zona glomerulosa hyperplasia in primary aldosteronism. Hypertension 56: 885-92.

13. Lau JGH, Sze WCC, Reznek RH, et al, 2012 A prospective evaluation of postural stimulation testing, computed tomography and adrenal vein sampling in the differential diagnosis of primary aldosteronism. Clin Endo 76: 182-188. 